Title

\title{
Cytoplasmic forces functionally reorganize nuclear condensates in oocytes
}

\section{Authors}

Adel Al Jord ${ }^{1 *}$, Gaëlle Letort ${ }^{1}$, Adrien Eichmuller ${ }^{1}$, Soline Chanet $^{1}$, Jean-René Huynh ${ }^{1}$, Nir S. Gov $^{2}$, Raphaël Voituriez ${ }^{3}$, Marie-Émilie Terret ${ }^{1}$, and Marie-Hélène Verlhac ${ }^{1}$

\section{Affiliations \& correspondence}

(1) Center for Interdisciplinary Research in Biology, Collège de France; UMR7241/U1050; PSL Research University, Paris 75005, France

(2) Department of Chemical and Biological Physics, Weizmann Institute of Science, Rehovot, 7610001, Israel

(3) Université Pierre et Marie Curie, UPMC, CNRS-UMR 7600 \& 8237; Sorbonne Universités, Paris 75005, France

(*) Corresponding author; email: adel.aljord@college-de-france.fr

\section{Running title}

Functional reorganization of nuclear condensates by cytoplasmic forces

\section{One sentence summary}

Cytoplasmic random forces in growing oocytes drive multiscale reorganization of nuclear liquid-like biomolecular condensates. 


\section{Abstract}

Cells remodel their cytoplasm with force-generating cytoskeletal motors ${ }^{1}$. Their activity generates random forces that stir the cytoplasm, agitating and displacing membranebound organelles like the nucleus in somatic $^{2-4}$ and $\mathrm{germ}^{5-7}$ cells. These forces are transmitted inside the nucleus ${ }^{4,7}$, yet their consequences on liquid-like biomolecular condensates $^{8-10}$ residing in the nucleus remain unexplored. Here, we probe experimentally and computationally diverse nuclear condensates, that include splicing speckles, Cajal bodies, and nucleoli, during cytoplasmic remodeling of female germ cells named oocytes. We discover that growing mammalian oocytes deploy cytoplasmic forces to timely impose multiscale reorganization of condensates inside the nucleus. We determine that cytoplasmic forces accelerate nuclear condensate collision-coalescence and molecular kinetics within condensates. Inversely, disrupting the forces decelerates nuclear condensate reorganization on both scales. We link the molecular deceleration found in mRNA-processing splicing speckles to reduced and altered splicing of mRNA, which in oocytes impedes fertility ${ }^{11}$. We establish that different sources of cytoplasmic forces can reorganize nuclear condensates and that this cytoplasmic aptitude for subnuclear reorganization is evolutionary conserved in insects. Our work implies that cells evolved a mechanism, based on cytoplasmic force tuning, to functionally regulate a broad range of nuclear condensates across scales. This finding opens new perspectives when studying condensate-associated pathologies like cancer, neurodegeneration and viral infections ${ }^{12}$.

\section{Main text}

Early embryogenesis depends on essential maternal transcripts and proteins accumulated during growth of developing female germ cells known as oocytes ${ }^{13}$. In mammals, growing oocytes remodel their cytoplasm to position the nucleus at the cell center despite undergoing an asymmetric division in size, which relies on chromosome off-centering, soon after ${ }^{14-16}$. Central nucleus position predicts

5 successful oocyte development in mice and humans ${ }^{14,15}$, and thus, their embryogenic potential. However, the spatiotemporal evolution and the purpose of this cytoplasmic remodeling with its consequent robust organelle positioning during mammalian oocyte growth remain unclear.

Cellular cytoplasmic remodeling is driven by cytoskeletal motor proteins ${ }^{1}$. The activity of motors generates random fluctuating forces that stir the whole cytoplasm, enhancing nonspecific transport of organelles and their agitation ${ }^{2-7}$. In growing mouse oocytes, cytoplasmic random stirring is predominantly driven by $\mathrm{F}$-actin and myosin motor-driven activity ${ }^{5}$ with physical consequences 
observable on two distinct spatiotemporal scales. On a large scale (hours to days), prolonged cytoplasmic random stirring displaces, nonspecifically, the large $30 \mu \mathrm{m}$ nucleus from the cell periphery to its geometrical center over a $25 \mu \mathrm{m}$ distance ${ }^{5,17}$. On a smaller scale (seconds), cytoplasmic random stirring agitates the nucleus with stochastic kicks, resulting in nuclear membrane fluctuations ${ }^{6,7}$. These cytoplasmic forces propagate inside the nucleus thus enhancing the mobility of the nucleolus ${ }^{7}$, one of many nuclear liquid-like compartments key for RNA processing and referred to as biomolecular condensates $^{8-10}$. We observed that, as growing mouse oocytes remodeled their cytoplasm (Fig.1A-C), the number of nucleoli decreased in favor of a size increase (Fig.1A-B orange arrowheads), as noted long ago in human oocytes ${ }^{18}$. Moreover, fully grown oocytes mutant for the F-actin nucleator Formin2 and consequent capacity to properly stir their cytoplasm (Fig.1A-C) presented a peripheral nucleus that was enriched in multiple immobile nucleoli of smaller sizes (Figs.1A-B orange arrowheads, S1A and $\left({ }^{7}\right)$ ). Since nucleoli as other liquid-like condensates can coalesce, this prompted us to investigate whether cytoplasmic forces during oocyte growth orchestrated the reorganization of liquid condensates inside the nucleus.

To proceed, we first revised an oocyte late-growth classification method, solely based on three easily identifiable features of chromatin condensation ${ }^{19}$ (Fig.1A), by linking it to a measure of cytoplasmic stirring and consequent nucleus position. Growing oocytes extracted from antral follicles presented the three expected chromatin states in a nucleus of constant volume (Fig.S1B-G). We found that smaller mid-antral "NSN" oocytes (№n-urrounded Nucleolus; decondensed interphase-like chromatin) had relatively low cytoplasmic F-actin (Fig.S1D, H) and stirring (Figs.1A-C, S1I, measured with image correlation analysis; Movie S1) with an off-centered poorly agitated nucleus (Fig.S1J), mirrored by moderate nucleus membrane fluctuations (Fig.S1K). Denser cytoplasmic F-actin (Fig.S1D, H) and stronger cytoplasmic stirring were quantified in subsequent bigger late-antral "Trans" oocytes (Transitioning pNSN; partially decondensed chromatin) that were in the process of nucleus centering (Figs.1A-C, S1 I-J). Comparably dense F-actin (Fig.S1D, H) and equally intense stirring were measured in the cytoplasm of fully grown "SN" oocytes (S cytoplasmic forces propagated onto a centrally positioned nucleus with a strongly fluctuating nuclear membrane (Figs.1A-C, S1I-K; Movie S1). Thus, oocytes maximally intensify cytoplasmic random stirring as of the early Trans growth-stage. This updated three-stage classification method, connecting known oocyte chromatin features with cytoplasmic stirring intensity, allowed us to inspect whether this cytoplasmic activity affects nuclear liquid condensates in growing oocytes.

To explore a global relation between cytoplasmic stirring and nuclear liquid condensates, we screened for multiple nuclear biomolecular condensates $^{9}$ in growing oocytes using known immunomarkers $^{20}$ of histone locus bodies (NPAT), paraspeckles (PSPC1), gems (SMN1), nucleoli (Fibrillarin), TDP-43 bodies (TDP-43), Cajal bodies (Coilin) and splicing speckles (SRSF2). Careful 3D-examination of these different condensates revealed their nuclear pattern evolution from the NSN to SN stages (Figs.1D, S2A-B). Further analyses demarcated condensates into two subpopulations with 
different properties. A first subpopulation of small shape-constrained condensates with stable chromatin association (Figs.S3A-B, S4A-B; in fuchsia), insensitivity to the droplet dissolver 1-6-hexanediol (Fig.S4C-E), and incomplete fluorescence recovery after photobleaching (Fig.S4F-H), collectively indicative of a non-liquid nature. A second subpopulation of large spherical and mobile droplet condensates that lacked apparent chromatin association (Figs.S3A-B, S4A-B; in orange), dissolved in response to 1-6-hexanediol (Fig.S4C-E), fully recovered fluorescence post-bleaching (Fig.S4F-H), and rapidly coalesced (Fig.S4I; Movies S2-S3), collectively indicative of liquid-like nature. We therefore focused on this second subpopulation of condensates to address our study's key question. We counted and measured sizes of various nuclear droplets in growing Control oocytes and in growing Formin2mutant oocytes $\left(\mathrm{FMN}^{-/}\right)$that present a significant drop in cytoplasmic stirring (Fig.S1I). In Controls, Coilin, SRSF2, nucleolar, and TDP-43 droplets decreased in number and increased in size as growth ended (Figs.1D-F, S2A, S5A-B). FMN2 ${ }^{-/}$oocytes, however, had more abundant yet smaller Coilin, SRSF2, nucleolar, and TDP-43 droplets as they reached the fully-grown stage (Fig.1E-F; Fig.S5A-C), despite Control-like total nuclear amounts of corresponding markers at tested growth stages (Fig.S6A). Other parameters known to modulate droplet size like cell and nucleus volume or neo-transcription were unaffected in mutants (Fig.S6B-C). Thus, the intensification of cytoplasmic random stirring in growing oocytes is linked to the spatiotemporal evolution of several subnuclear liquid condensates, potentially through increased droplet coalescence dynamics.

To confirm that cytoplasmic stirring enhanced nuclear droplet dynamics, we live-imaged at high temporal resolution growing oocytes microinjected with RNA encoding the splicing speckle marker SRSF2-GFP. We chose SRSF2 due to droplet abundance at all stages of growth (Figs.S2A-B, S5A) and verified that SRSF2-GFP expression profiles were comparable to endogenous SRSF2. In all three stages of growth, we observed local droplet displacements instigated by the fluctuating nuclear membrane (Figs.2A, S7A; Movie S3), an immediate consequence of cytoplasmic stirring forces agitating the nucleus ${ }^{7}$. Locally pushed droplets collided and fused with neighboring droplets when encountered (Fig.2A; Movie S3). Droplet diffusion, integrating both local and global cytoplasmic forces acting on the nucleus, was inversely proportional to its radius (Fig.S7B) and correlated positively with the intensification of cytoplasmic forces during growth (Fig.S7C). This was consistent with the hypothesis that cytoplasmic forces induce active diffusion of intranuclear droplets (see Biophysical model in Methods). We therefore tuned the transmission of cytoplasmic forces to the nucleoplasm, using established pharmacological and genetic tools ${ }^{5,7}$ (Figs.2B, S7D), before monitoring droplet diffusion at distinct growth stages (Figs.2C, S7E; Movie S4). Amplifying nucleus agitation by disrupting forcedampening perinuclear microtubules with Nocodazole (as in $\left({ }^{7}\right)$ ) increased the effective diffusion of droplets at all stages when compared to Controls (Figs.2C, S7E; Movie S4). Inversely, obstructing forces from the nucleus by Taxol-mediated stabilization of microtubules slowed down droplets (Figs.2C, S7E; Movie S4). Greater deceleration occurred in cells with disrupted cytoplasmic actomyosin-based forces due to Cytochalasin-D incubation or FMN2 knockout (Figs.2C, S7E; Movie S4). Depleting residual microtubule-based forces in FMN2 $2^{-/}$oocytes with Nocodazole further diminished nuclear droplet 
diffusion (Fig. S7E). Droplet diffusion generally remained sub-diffusive (Fig.S7E right panel; $\alpha<1$ ), consistent with chromatin-mediated constraints on droplet diffusion ${ }^{21}$. Thus, cytoplasmic forces, by agitating the nucleus, enhance diffusive dynamics of nuclear droplets in constrained environments. Moreover, tuning nucleus agitation led to droplet size changes matching agitation intensity without affecting droplet shape or total SRSF2-GFP expression levels (Fig.S7F-G). Altogether, this suggested that cytoplasmic stirring, by enhancing droplet diffusivity, favored nuclear droplet encounters and coalescence.

To verify the cytoplasmic drive of nuclear droplet coalescence, we documented longer-term consequences of cytoplasmic stirring forces on nuclear droplet dynamics. The 3D-tracking revealed stochastic displacements of droplets (Figs.2D-E) that started bouncing randomly around the nucleoplasm more intensely and frequently as of the Trans-stage (Figs.2E, S8A) which is concomitant to cytoplasmic stirring intensification (Fig.S1I). Droplets eventually encountered one another, collided and fused (Figs.2F, S8B; Movie S5). In FMN2 mutants, droplets bounced less and less frequently (Figs.2D-E, S8A), and the frequency decreased further when both F-actin and microtubules were disrupted (Fig.S8A, C-D). We therefore modulated cytoplasmic forces and measured nuclear droplet coalescence kinetics in NSN oocytes that harbor significantly more droplets than SN counterparts (Fig.2G, S8E-G; Movie S6). Amplifying cytoplasm-based nucleus agitation accelerated droplet coalescence, manifested by a quicker decline in droplet number coupled to droplet size increase when compared to Controls (Fig.2G). Inversely, taming nucleus agitation decelerated droplet coalescence, revealed by weak droplet number decline and mean droplet size changes within the 100-minute filming period (Fig.2G). Chromatin remained in a decondensed state in analyzed cells (Fig.S8G), hence eliminating the chromatin condensation bias potentially impacting droplet coalescence. Thus, cytoplasm-based agitation of the nucleus accelerates nuclear droplet collision-coalescence kinetics. To further dissect the cytoplasmic drive of nuclear droplet collision-coalescence, we built an agent-based model of SRSF2-like droplet diffusion in a nucleus-like spherical container agitated by cytoplasmic forces (see Methods). Nuclear obstacles, based on experimental chromatin surface measurements, were added to enforce sub-diffusive motion thus mimicking nuclear crowding by chromatin. 3D-numerical simulations were calibrated to recapitulate Control-NSN nuclear droplet diffusive dynamics on the seconds timescale (Fig.S9A). Running these simulations on 100-minute timescales reproduced experimental Control and modulated cytoplasmic drives of nuclear droplet coalescence in NSN oocytes (Fig.2H for droplet coalescence speed; Fig.S9B for droplet number and size changes). Thus, nuclear droplet diffusion and collision-coalescence propelled by cytoplasmic forces are sufficient to explain the observed droplet coarsening in the crowded nucleus.

To estimate the minimal time necessary to reorganize nuclear compartments if cells were to transition from NSN to SN states of oocyte growth, a process occurring inside the follicle that cannot be followed in live, and to evaluate chromatin's interference with the process, we ran our computational model on longer timescales with wider ranges of starting-point cytoplasmic force intensities (Figs.2I-J, S9C-E). 
To mimic key physiological cytoplasmic and nucleoplasmic events that initiate at the end of oocyte growth, we incorporated into our simulations cytoplasmic force intensification as well as chromatin condensation (Fig. S9C simulation series 2 with transition at 12 hours; see Methods) that naturally occur after the NSN-stage (Fig.S1B-C, I). Simulations revealed a stimulus-response relationship between the cytoplasm and nucleoplasm, whereby the intensity of cytoplasmic forces defined the speed of nuclear SRSF2 droplet collision-coalescence (Fig.2I-J; Movie S7). To reach the endogenous compartmentalization state of 4 nuclear SRSF2 droplets observed at the final growth stage (mean SRSF2 droplet number in Trans cells with a central nucleus), Control oocytes would require $15 \pm 8$ hours from the NSN|Trans transition (Figs.2I-J, S9E Series 2; Movie S7). This prediction is consistent with in vivo mid-to-end of antral oocyte growth, a process roughly estimated to occur within less than 2 days physiologically. The prediction is also consistent with prior experimental in vitro and computational studies since it falls within the established minimal time-range of 5 to 17 hours necessary for cytoplasmic activity to displace the peripheral nucleus to the cell center ${ }^{5,17}$. In contrast, cells with diminished cytoplasmic forces insufficient to transport the nucleus, such as FMN2 $2^{-/}$oocytes ${ }^{5,17}$, would require $59 \pm 8$ hours to reach a nuclear compartmentalization state comparable to Controls (Figs.2I-J, S9E Series 2; Movie S7). In addition to the importance of nucleus agitation, these simulations highlighted chromatin condensation-based acceleration of droplet fusion dynamics (compare before|after $\mathrm{t}=12 \mathrm{~h}$ in Fig.2I). We therefore generated two supplementary models to further weigh chromatin's interference with nuclear droplet fusion kinetics (Fig.S9C-F; series $1 \& 3$ ). We modelled an NSN nucleus state with interphaselike decondensed chromatin and a SN nucleus state with condensed chromatin before launching the simulations with wide ranges of cytoplasmic force intensities, maintained constant in time. Droplet collision-coalescence was faster in the condensed chromatin set-up (Series 3 ) for all cytoplasmic force intensities (Fig.S9D), suggesting a kinetic barrier effect for chromatin that is consistent with its solidlike and diffusion-constraining properties ${ }^{21,22}$. Probing further, we found that with weak cytoplasmic forces (FMN2 $2^{-/}$-like scenario), chromatin significantly hindered droplet fusion kinetics, seen by the substantial drop in time required to coalesce all droplets post-chromatin condensation (Fig.S9F; compare $\mathrm{FMN}^{-/}$plain and dashed fits). In all Control-like scenarios, however, cytoplasmic forces significantly reduced the chromatin's drag of droplet fusion (Fig.S9F; compare NSN, Trans, and SN plain and dashed fits). Thus, the computational models anticipate cytoplasmic force intensification to be a predominant driver of nuclear droplet coalescence in physiological contexts. Fixed and live experiments supported by computational modeling indicate that cytoplasmic forces in growing oocytes spatially reorganize subnuclear liquid compartments by boosting large-scale droplet collision-coalescence.

To determine local sub-droplet-scale consequences of cyto-to-nucleoplasmic force transfer, we first measured rapid surface fluctuations of large SRSF2-GFP droplets in Control and F-actin mutant SN oocytes \pm Nocodazole. Surface fluctuations of comparably sized droplets declined when F-actin-based cytoplasmic forces were disrupted, and decreased further after simultaneous disruption of both F-actin and microtubules (Fig.3A, S10A). The Control:mutant droplet fluctuation ratio (2) was smaller than the Control:mutant nuclear membrane fluctuation ratio $\left(\cong 6\right.$ in $\left({ }^{7}\right)$ ), consistent with expected energy 
dissipation theorized by our biophysical model that links cytoplasmic stirring with the nucleoplasm (see Methods). The observable transfer of cytoplasmic forces onto nuclear droplets suggested the enhancement of droplets' internal molecular kinetics that we subsequently measured using FRAP. Droplet molecules diffused significantly slower in $\mathrm{FMN}^{-{ }^{--}}$cells and cells treated with Cytochalasin-D than in Controls (Figs.3B-C, S10B-D). Molecular dynamics in droplets were reversible after restoration of cytoplasmic stirring by drug washout (Figs.3C, S10B-D). Thus, cytoplasm-based agitation of the nucleus enhances nuclear sub-droplet-scale kinetics by amplifying droplet surface fluctuations and interior molecular mobility.

Biomolecule mobility in condensates is expected to boost localized biochemical reactions, which in splicing speckles correspond to mRNA processing by the spliceosome ${ }^{23}$. RNA-binding SR proteins like SRSF2 generally activate or alter splicing by binding specific regions of pre-mRNA known as exon splicing enhancers ${ }^{24}$. We therefore hypothesized that cytoplasmic forces, by accelerating molecular diffusion in droplets, would enhance protein-RNA interaction kinetics that activate or alter splicing. To first test the splicing activity hypothesis, we probed catalytically active spliceosomes engaged in mRNA splicing using a specific phospho-marker (pThr313-SF3b155) $\left({ }^{23}\right)$ during oocyte growth \pm cytoplasmic stirring. Consistent with the general transcriptional decline during Control and mutant oocyte growth (Fig.S6B-C), total nucleoplasmic splicing activity decreased (Figs.3D-E, S11A) while total SF3b155 levels remained comparable (Fig.S11B). Despite the major drop in transcription during the NSN to SN transition (Fig.S6B-C), active splicing in SRSF2 ${ }^{+}$droplets remained unexpectedly constant in growing Control oocytes (Fig.3D-E left, S11A). In FMN2 ${ }^{-/-}$oocyte droplets, however, active splicing significantly dropped as of the Trans-stage (Fig.3D-E right, S11A), which is when cytoplasmic stirring intensified in Controls (Fig. S1I). This suggested that cytoplasmic force intensification sustains active splicing in nuclear droplets. To next test the alternative splicing hypothesis, we performed splicingcentered bioinformatic analyses of validated mRNA-sequencing data comparing Control with mutant SN oocytes ${ }^{7}$, the stage during which the observed splicing decline was maximal (Fig.3D-E). We detected thousands of exon usage differences in mutant oocytes with 1785 overrepresented exons that were in average significantly shorter than the 948 underrepresented exons (Figs.3F, S11C-D; Table S1), suggesting alternative splicing with enrichment of short exon inclusion and long exon skipping. To probe the mRNA processing imbalance further, we scrutinized differential transcript isoform usage, a direct consequence of alternative splicing, and uncovered hundreds of isoform switches in mutant oocytes (Figs.3G, S11E-H; Table S2). Multiple differential splicing events per transcript were identified (Fig.S11F) for a sum of 3565 alterations in 1259 transcripts (Fig.S11G), ranging from splice site selection to exon skipping and intron retention, with 2178 predicted consequences on isoform length or stability (Fig.S11H). We confirmed the tendencies of some differential exon usage and splicing outcomes with RT-qPCR (Fig.S11C, E). Thus, splicing activity is altered in growing oocytes with insufficient cytoplasmic forces. Consistent with our hypothesis, these data therefore imply that cytoplasmic forces in oocytes alter overall mRNA processing with broad consequences on maternal reservoir transcripts, the quality of which is key for successful embryogenesis. 
We finally assessed evolutionary conservation of the cytoplasmic stirring's capacity to reorganize subnuclear liquid compartments. We chose Drosophila melanogaster oocytes due to their well described cytoplasmic stirring dynamics ${ }^{25,26}$, yet whose consequences on the nucleus interior remain unexplored. We therefore immunostained the splicing speckle marker SC35 (mouse SRSF2 homologue) in mid-oogenesis Drosophila oocytes (stage 9) when the cytoplasm is randomly stirred by microtubules and buffered by an actin mesh ${ }^{25,26}$, which notably is the exact opposite of what occurs in mouse oocytes where microtubules buffer actin-based stirring of the cytoplasm ${ }^{5,7}$. Disrupting the buffering actin mesh precociously via genetic inhibition of two distinct F-actin nucleators Cappuccino or Spire (mouse FMN2 or SPIRE homologues), amplified microtubule-based stirring of the cytoplasm and prematurely induced fast streaming (Fig.4A-B), a known transporting mechanism that, in various animals, plants and fungi, is vital for reproduction or developmental growth and differentiation ${ }^{25,27}$. Premature fast streaming during growth consistently led to larger yet less numerous nuclear condensates by stage 9 when compared to Controls (Fig.4C-E). Inversely, stabilizing the actin mesh by overexpressing a constitutively active Spire blocked cytoplasmic stirring in growing oocytes ${ }^{25}$ that, by stage 9, coherently lacked large nuclear condensates and showed enrichment of smaller condensates (Fig.4A-E). Thus, cytoplasmic actin-based random forces, as in mouse oocytes, or microtubule-based random or constant forces, as in fly oocytes, can boost subnuclear condensate fusion. Moreover, the aptitude of cytoplasmic stirring to refashion nuclear liquid compartments is evolutionary conserved and could potentially be deployed by other distant species for similar purposes.

This multidisciplinary study uncovers that growing mouse oocytes remodel their cytoplasm to enhance nuclear biomolecular condensate kinetics concurrently across scales (Fig.S12). The increase in cytoplasmic stirring forces functions simultaneously as a nuclear condensate collider, boosting mesoscale reorganization in the nucleus, and as a condensate core particle accelerator, altering molecular-scale reactions associated with the biomolecular condensate's function like mRNA processing. In the case of oocytes, which become nutritional vessels for early embryos ${ }^{13}$, transcript processing quality is vital for sustainability of ensuing embryogenesis ${ }^{11}$. The scale-crossing kinetic link between cytoplasmic remodeling and functional nuclear droplet reorganization shown here sheds light on why cytoplasmic remodeling and its consequent nucleus positioning in mammalian oocytes is such a consistent predictor of their embryogenic potential ${ }^{14,15}$. This finding may therefore guide future frameworks of reproductive efforts that include human in vitro fertilization and oocyte somatic cell nucleus transfer ${ }^{28}$ for agricultural reproductive cloning or endangered species conservation.

We report that growing cells intensify cytoskeletal stochastic forces in the cytoplasm to achieve timely spatial reorganization of diverse liquid condensates inside the nucleus. The necessity for this cytoplasmic active force increase may be explained by the crowded nature of the nucleus, substantially filled with solid-like and diffusion-constraining chromatin ${ }^{21,22}$ acting as a barrier to the reorganization kinetics of nuclear condensates. We also show that these same forces modulate splicing speckle- 
associated mRNA processing and this is likely to be relevant to other RNA-processing condensates present in the nucleoplasm. This is pertinent for tumor-related studies since human malignant cancer cells, in opposition to benign ones, were shown to intensify cytoplasmic random forces ${ }^{2}$. We speculate that the exploitation of these forces could enhance RNA-processing activity associated with various nuclear condensates to sustain high metabolic needs of cells in a growing tumor. Together, our study reveals the physiological existence of a coordinating cellular mechanism that couples spatial reorganization of condensates at the mesoscale with functional molecular-scale changes within the same condensates, thus providing a concrete mechanistic example to recent speculations linking different condensate-associated length scales ${ }^{10}$.

Conceptually, our finding is consistent with the ability of evolutionary distant organisms to exploit cellscale cytoskeletal stochasticity for ordered changes in tissue-scale organization during development ${ }^{29,30}$. Like a miniature tissue, individual cells can thus physiologically calibrate and deploy a nondeterministic physical input, taking the form of cytoplasmic random forces, to generate orderly intracellular outputs, marked by complex subnuclear reorganization of liquid condensates and associated optimal biochemical activity tailored to cell fate. This cellular mechanism based on rapid stochastic force tuning may prove to be a common regulator of intranuclear kinetics of liquid-like condensates, which are inherent to prompt adaptive responses to sudden environmental changes ${ }^{8-10}$. 


\section{References}

290 1. Pegoraro, A. F., Janmey, P. \& Weitz, D. A. Mechanical properties of the cytoskeleton and cells. Cold Spring Harb. Perspect. Biol. 9, a022038 (2017).

2. Guo, M. et al. Probing the Stochastic, Motor-Driven Properties of the Cytoplasm Using Force Spectrum Microscopy. Cell 158, 822-832 (2014).

3. Fakhri, N. et al. High-resolution mapping of intracellular fluctuations using carbon nanotubes. Science 344, 1031-1035 (2014).

4. $\quad$ Chu, F. Y., Haley, S. C. \& Zidovska, A. On the origin of shape fluctuations of the cell nucleus. Proc. Natl. Acad. Sci. U. S. A. 114, 10338-10343 (2017).

5. Almonacid, M. et al. Active diffusion positions the nucleus in mouse oocytes. Nat. Cell Biol. 17, 470-479 (2015).

300 6. Ahmed, W. W. et al. Active Mechanics Reveal Molecular-Scale Force Kinetics in Living Oocytes. Biophys. J. 114, 1667-1679 (2018).

7. Almonacid, M. et al. Active Fluctuations of the Nuclear Envelope Shape the Transcriptional Dynamics in Oocytes. Dev. Cell 51, 145-157 (2019).

8. Shin, Y. \& Brangwynne, C. P. Liquid phase condensation in cell physiology and disease. Science 357, 1-11 (2017).

9. Banani, S. F., Lee, H. O., Hyman, A. A. \& Rosen, M. K. Biomolecular condensates: Organizers of cellular biochemistry. Nat. Rev. Mol. Cell Biol. 18, 285-298 (2017).

10. Lyon, A. S., Peeples, W. B. \& Rosen, M. K. A framework for understanding the functions of biomolecular condensates across scales. Nat. Rev. Mol. Cell Biol. (2020). doi:10.1038/s41580020-00303-z

11. Do, D. V. et al. SRSF3 maintains transcriptome integrity in oocytes by regulation of alternative splicing and transposable elements. Cell Discov. 4, 1-16 (2018).

12. Alberti, S. \& Dormann, D. Liquid - Liquid Phase Separation in Disease. Annu. Rev. Genet. 53, 171-196 (2019).

315 13. Clift, D. \& Schuh, M. Restarting life: fertilization and the transition from meiosis to mitosis. Nat. Rev. Mol. Cell Biol. 14, 549 (2013).

14. Brunet, S. \& Maro, B. Germinal vesicle position and meiotic maturation in mouse oocyte. Reproduction 133, 1069-1072 (2007).

15. Levi, M., Ghetler, Y., Shulman, A. \& Shalgi, R. Morphological and molecular markers are correlated with maturation- competence of human oocytes. 28, 2482-2489 (2013).

16. Binyam, M., Scheffler, K. \& Schuh, M. Assembly and Positioning of the Oocyte Meiotic Spindle. Annu. Rev. Cell Dev. Biol. 34, 381-403 (2018).

17. Colin, A. et al. Active diffusion in oocytes nonspecifically centers large objects during prophase I and meiosis I. J. Cell Biol. 219, e201908195 (2020).

325 18. Miyara, F. et al. Chromatin configuration and transcriptional control in human and mouse oocytes. Mol. Reprod. Dev. 64, 458-470 (2003).

19. Bonnet-Garnier, A. et al. Genome organization and epigenetic marks in mouse germinal vesicle oocytes. Int. J. Dev. Biol. 56, 877-887 (2012).

20. Sawyer, I. A., Sturgill, D. \& Dundr, M. Membraneless nuclear organelles and the search for phases within phases. Wiley Interdiscip. Rev. RNA 10, 1-20 (2019).

21. Lee, D. S. W., Wingreen, N. S. \& Brangwynne, C. P. Chromatin mechanics dictates subdiffusion and coarsening dynamics of embedded condensates. Nat. Phys. (2021). doi:10.1038/s41567-02001125-8

22. Strickfaden, H. et al. Condensed Chromatin Behaves like a Solid on the Mesoscale In Vitro and in Living Cells. Cell 183, 1772-1784.e13 (2020).

23. Girard, C. et al. Post-transcriptional spliceosomes are retained in nuclear speckles until splicing completion. Nat. Commun. 3, 994 (2012).

24. Nilsen, T. W. \& Graveley, B. R. Expansion of the eukaryotic proteome by alternative splicing. Nature 463, 457-463 (2010).

340 25. Quinlan, M. E. Cytoplasmic Streaming in the Drosophila Oocyte. Annu. Rev. Cell Dev. Biol. 32, 173-195 (2016). 
26. Drechsler, M., Giavazzi, F., Cerbino, R. \& Palacios, I. M. Active diffusion and advection in Drosophila oocytes result from the interplay of actin and microtubules. Nat. Commun. 8, 1520 (2017).

27. Goldstein, R. E. \& van de Meent, J. W. A physical perspective on cytoplasmic streaming. Interface Focus 5, 20150030 (2015).

28. Gurdon, J. B. \& Wilmut, I. Nuclear transfer to eggs and oocytes. Cold Spring Harb. Perspect. Biol. 3, a002659 (2011).

29. Martin, A. C., Kaschube, M. \& Wieschaus, E. F. Pulsed contractions of an actin-myosin network drive apical constriction. Nature 457, 495-499 (2009).

30. Rauzi, M., Lenne, P. F. \& Lecuit, T. Planar polarized actomyosin contractile flows control epithelial junction remodelling. Nature 468, 1110-1115 (2010).

\section{End Notes}

\section{Acknowledgements}

We thank T. Lecuit, V. Lallemand-Breitenbach, A. Meunier, M. Piel, and F.C. Tsai for comments on the manuscript; E. Batsché, G. Cecere, M. Singh, and all past and present members of the Verlhac-Terret laboratory for discussions; E. Anceaume, J. Dumont, P. Mailly, and T. Piolot from the CIRB microscopy platform for imaging support; the CIRB animal facility for animal care; N. Braure, S. Grosclaude, N.G. Kouakou, and S. Maussion for administrative support; C. Antoniewski, L. Bellenger, N. Naouar, and M. Raymond from the IBPS ARTbio bioinformatics platform for their services; M. Ducom (www.marieducom.com) for designing all illustrations following discussions with A.A.J..

\section{Funding}

365 The Verlhac-Terret and Huynh laboratories are supported by CNRS, INSERM, College de France and the Bettencourt Schueller Foundation. This work received support under the program « Investissements d'Avenir » launched by the French Government and implemented by the ANR, with the references: ANR-10-LABX-54 MEMO LIFE, ANR-11-IDEX-0001-02 PSL* Research University; and through grants from the Fondation pour la Recherche Médicale (FRM label to MHV DEQ201903007796; J.R.H. DEQ20160334884), and the ANR (ANR-18-CE13 to M.H.V.; ANR-16-CE13 to M.E.T.; ANR-15CE13-0001-01 to J.R.H.). S.C. received an ARC fellowship. N.S.G. is the incumbent of the Lee and William Abramowitz Professorial Chair of Biophysics. A.A.J. received fellowships from ARC (PDF2017050561) and Labex Memolife 2.0.

\section{Author contributions}

A.A.J. and M.H.V. conceived the project, which was supervised by M.E.T. and M.H.V. A.A.J. designed and performed all experiments on mouse oocytes with assistance from A.E and M.H.V. for microinjections. S.C. and J.R.H. did all Drosophila oocytes experiments. A.A.J. analyzed all mouse and Drosophila experiments. G.L. performed 3D-computer simulations. A.A.J. directed the analysis of the RNA-seq data in collaboration with the IBPS ARTbio bioinformatics platform. N.S.G. and R.V. contributed with the physical modeling and participated in discussions on data interpretations. A.A.J. wrote the manuscript, which was seen and corrected by all authors.

\section{Competing interests}

The authors declare no competing interests. 


\section{Data \& materials availability}

All data are in the manuscript and in the Extended Data.

390

\section{Code availability}

Code description and links are in the Methods.

\section{Correspondence}

395 To Adel Al Jord (adel.aljord@college-de-france.fr)

\section{Keywords}

Oocyte; Growth; Nucleus; Cytoplasmic stirring; Cytoskeleton; Force fluctuations; Actin; Microtubules; Biomolecular condensates; Liquid-liquid phase separation; Cell compartments; Nucleome.

400

\section{Extended Data include:}

Materials and Methods

Figures $\mathrm{S} 1$ to $\mathrm{S} 12$

Captions for Figures S1 to S12

405

Captions for Movies S1 to S7

Captions for Tables S1 to S2

Supplementary references

Separate files: Movies S1 to S7 and Tables S1 to S2 
bioRxiv preprint doi: https://doi.org/10.1101/2021.03.15.434387; this version posted March 16, 2021. The copyright holder for this preprint (which was not certified by peer review) is the author/funder. All rights reserved. No reuse allowed without permission.

Figure 1

A

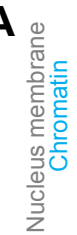
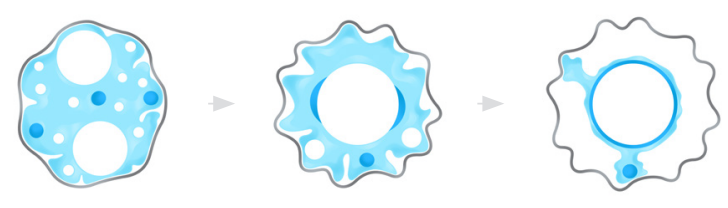

B
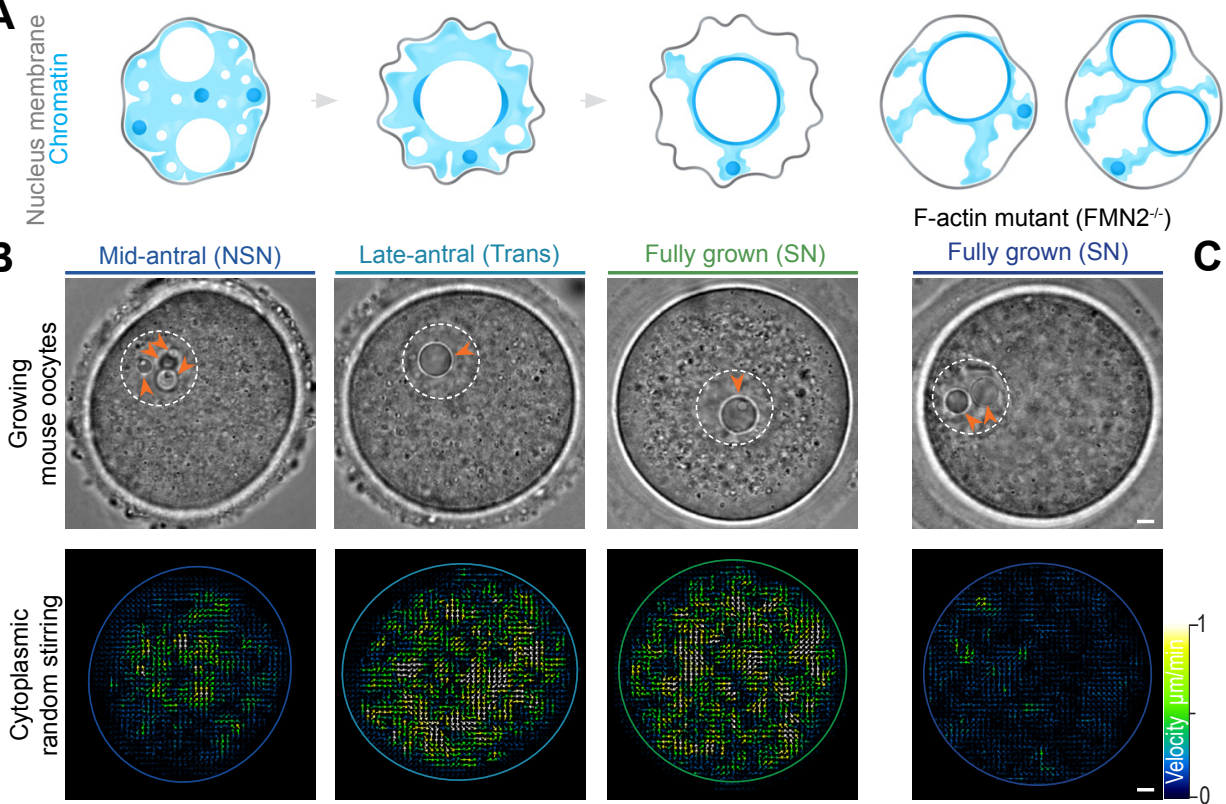

F-actin mutant $\left(\mathrm{FMN}^{-/-}\right)$

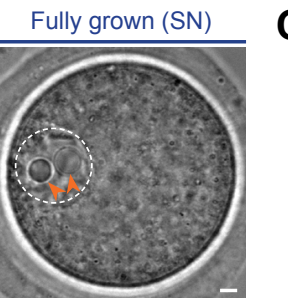

C
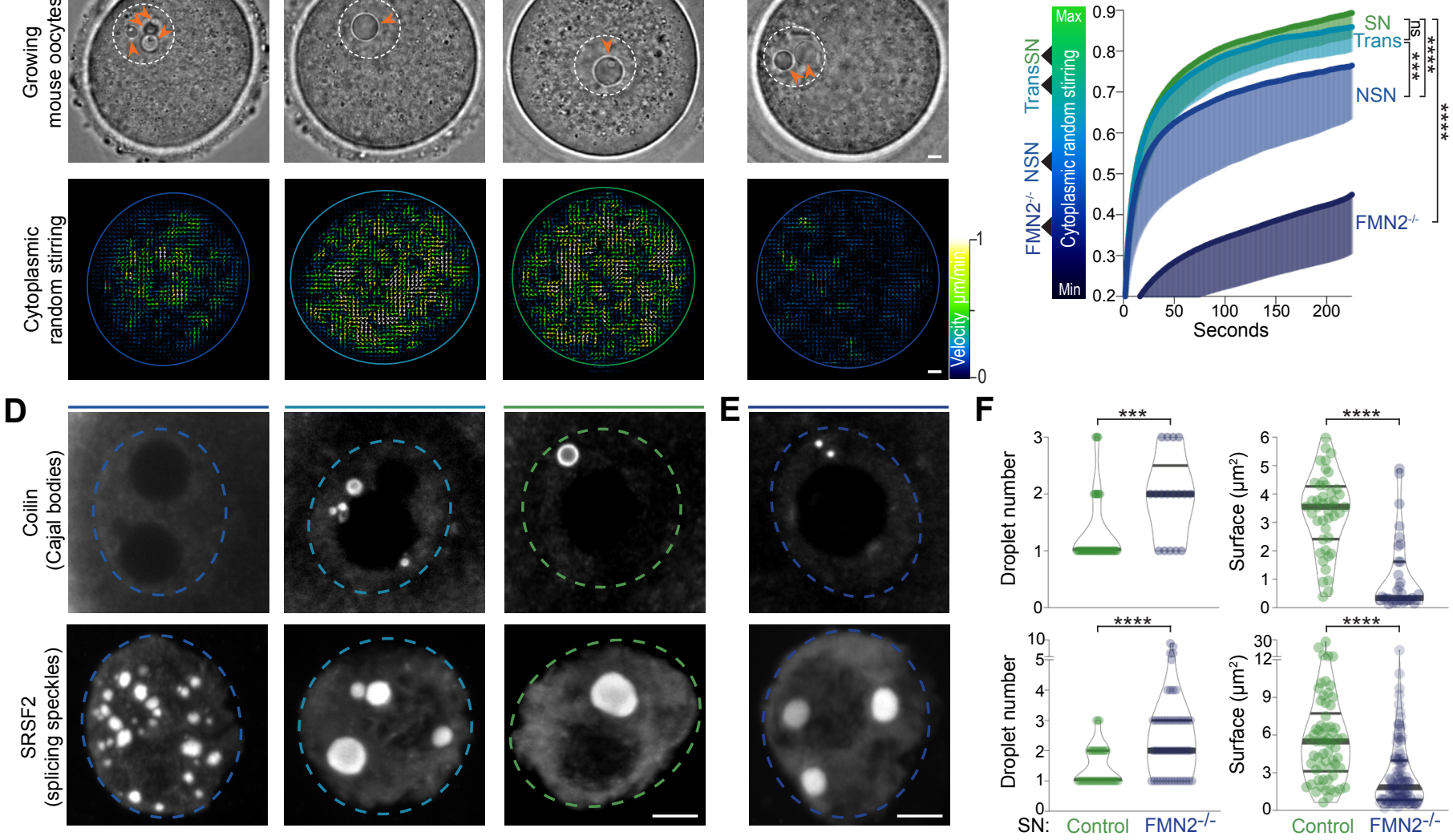
Fig. 1 | Nuclear biomolecular condensate patterns during mouse oocyte growth correlate with cytoplasmic remodeling

(A) Illustration of chromatin configuration evolution (NSN-Trans-SN) coinciding with oocyte consecutive growth subcategories (mid-antral follicle, late-antral follicle, and fully grown); (left) large spherical nucleoli decrease in number in favor of a size increase as of the Trans-stage; (right) chromatin configuration scenarios in fully grown cytoplasmic F-actin mutants with one large or two smaller nucleoli. The nuclear membrane (gray) is depicted according to its fluctuation intensity at distinct stages of growth (see $\left(^{7}\right)$ and Fig.S1I). (B) Top: Bright-field images of (left panels) Control oocytes with growth progression and (right panel) a fully grown mutant; the nucleus is outlined in dashed white and multiple small nucleoli (NSN and $\mathrm{FMN}^{-/-} \mathrm{SN}$ ) or a single large nucleolus (Trans and $\mathrm{SN}$ ) are indicated with orange arrowheads. Bottom: Growth-associated cytoplasmic stirring vector maps generated by STICS analyses of bright-field 240 seconds-stream videos; the oocyte cortex is outlined with colors reflecting the intensity of cytoplasmic stirring; maps are color coded according to velocity magnitude (lowest in dark blue, strongest in white). (C) Cytoplasmic random stirring intensity in time measured in Control NSN to SN and FMN2 ${ }^{-/-}$SN oocytes by image correlation analyses of cytoplasmic pixel evolution; color gradient (left) used in this study to represent the cytoplasmic stirring intensity; cell number, Control $\mathrm{NSN}=22$, Trans $=19, \mathrm{SN}=28$ cells, $\mathrm{FMN} 2^{-/} \mathrm{SN}=13$; error bars represent mean-s.d.; $P$ values derived from two-tailed Mann-Whitney $U$-Tests on plateau distributions, ns, not significant, $P=0.074$, $* * * P=0.0001, * * * * P<0.0001$. (D-E) Representative immunoreactivities of Coilin and SRSF2 (a.k.a. $\mathrm{SC} 35$ ) in growing Control oocytes and SN FMN2 ${ }^{-/}$oocytes; Coilin images are $0.5 \mu \mathrm{m}$ z-planes and SRSF2 images are $20 \mu \mathrm{m}$ z-projections; nucleus regions outlined with dashed circles. (F) Quantifications of Coilin (top) and SRSF2 (bottom) droplet number and surface in nuclei of fully grown SN Control and mutant oocytes; Coilin, counted in 43 Control and $17 \mathrm{FMN}^{-/-}$cells, measured 44 Control and $33 \mathrm{FMN} 2^{-}$ ${ }^{\prime-}$ droplets; SRSF2, counted in 41 Control and $52 \mathrm{FMN}^{-{ }^{--}}$cells, measured 62 Control and $112 \mathrm{FMN}^{-/-}$ droplets; violin plots with median \pm quartiles; $P$ values derived from two-tailed Mann-Whitney $U$-Tests, $* * * P=0.0004, * * * * P<0.0001$. Color codes based on cytoplasmic stirring intensities; scale bars, $5 \mu \mathrm{m}$. 
bioRxiv preprint doi: https://doi.org/10.1101/2021.03.15.434387; this version posted March 16, 2021. The copyright holder for this preprint (which was not certified by peer review) is the author/funder. All rights reserved. No reuse allowed without permission.

Figure 2

\section{A}

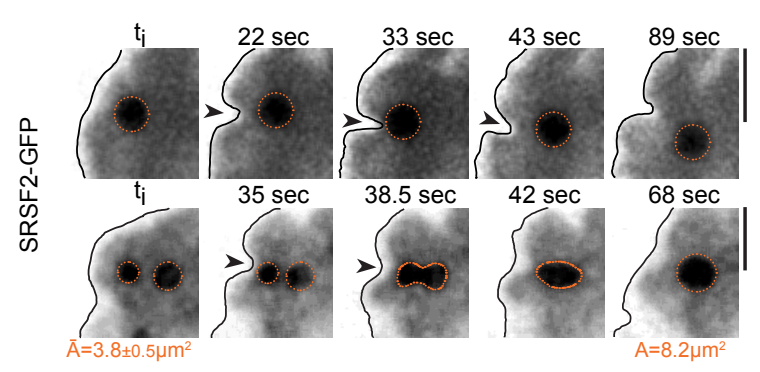

D

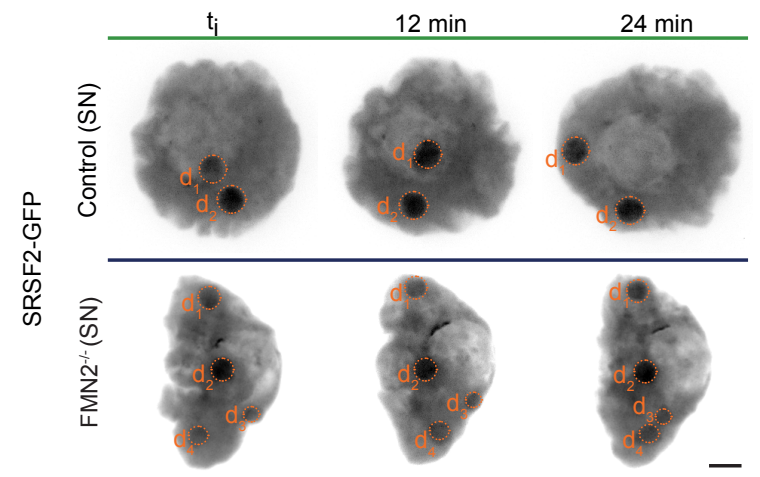

B

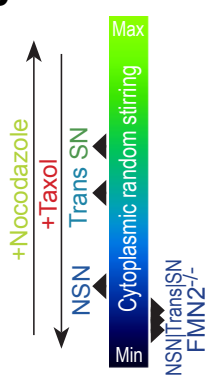

C

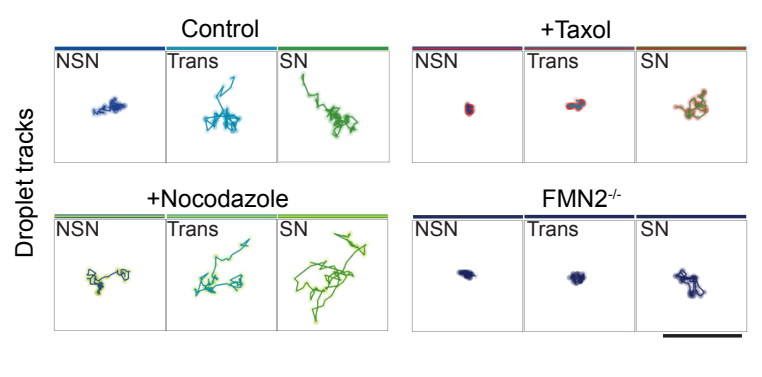

$\mathbf{E}$

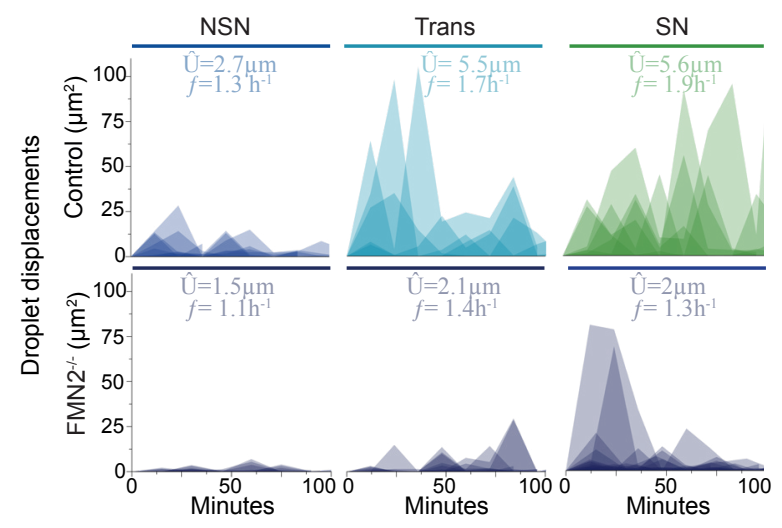

$\mathbf{F}$
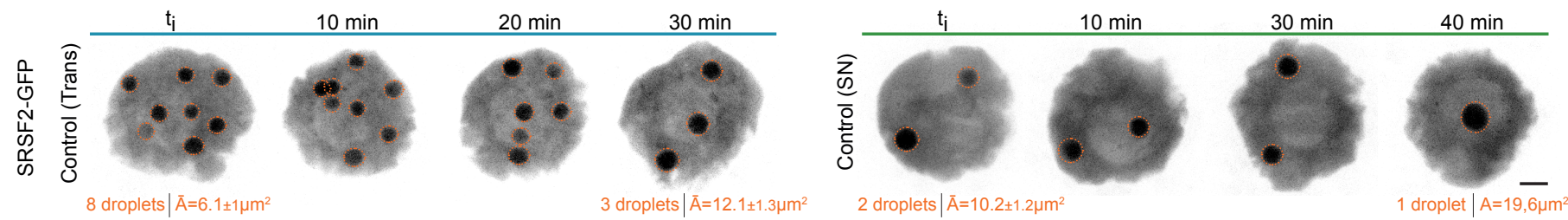

G

H
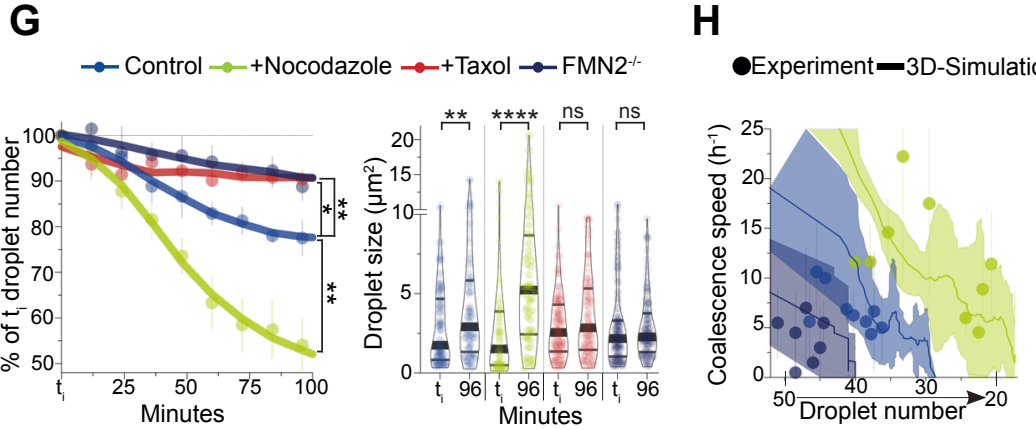

I
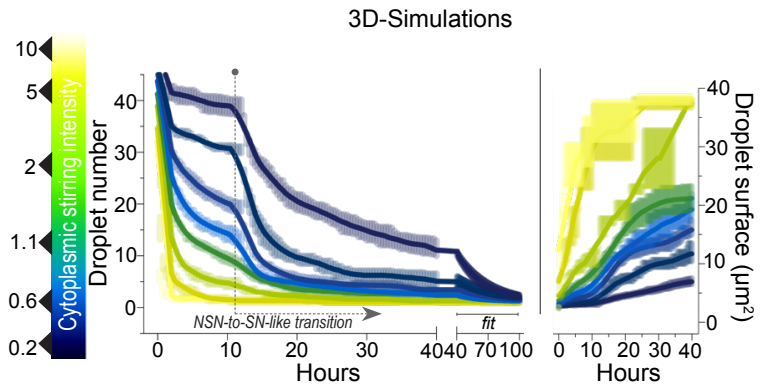

J

NucleusSRSF2 droplets Nucleolus

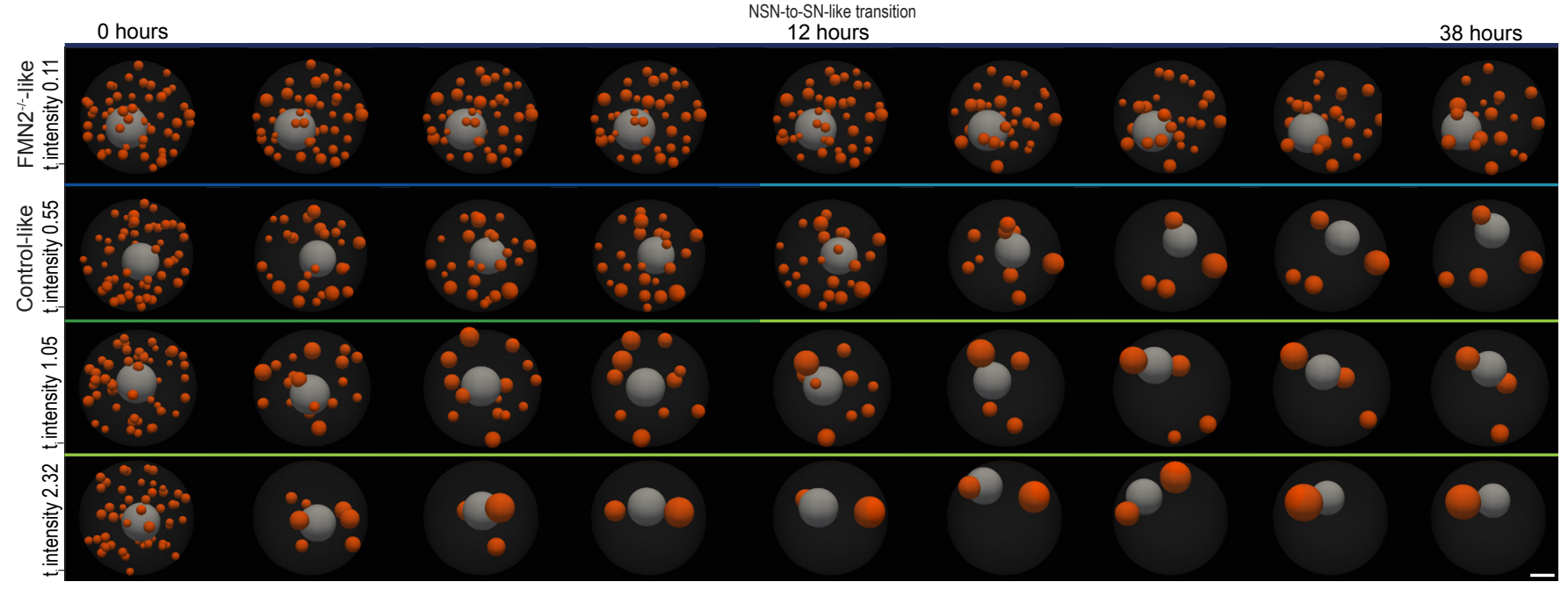


Fig. 2 | Cytoplasmic random forces drive micron-scale liquid condensate collision-coalescence in the nucleus

410 (A) Time-lapse 2D-images showing nuclear membrane invaginations physically kicking and locally displacing nucleoplasmic SRSF2-GFP droplets (seconds timescale) in Trans (top) or NSN (bottom) cells, directly causing droplet collision-coalescence; black arrowheads indicate nuclear membrane invaginations and the membrane is outlined in black. (B) Color gradient used in this study to represent the intensity of cytoplasmic forces in growing Control and mutant oocytes or Controls treated with microtubule inhibitors; Nocodazole disrupts perinuclear force-dampening by microtubules thus intensifying force transmission, whereas Taxol stabilizes perinuclear microtubules, obstructing cytoplasmic forces from the nucleus. (C) Representative SRSF2-GFP droplet 2D-space exploration on a seconds-timescale (50 second-tracks) at the three growth stages in Control oocytes, Controls incubated with Nocodazole or Taxol, and FMN2 $2^{-/}$oocytes. (D) Time-lapse $50 \mu \mathrm{m}$ z-projections showing minutetimescale 3D-displacements of tracked SRSF2-GFP droplets in the nucleoplasm of fully-grown SN Control and FMN2 ${ }^{-/}$oocytes. (E) 3D-tracks of SRSF2-GFP droplets revealing fluctuating displacements (squared) in Control and $\mathrm{FMN}^{-/-}$oocytes at three stages of growth; droplet displacement amplitude $\hat{\mathrm{U}}$ and frequency $f$ depicted above tracks; droplet number in Control, NSN $=8$, Trans $=5, \mathrm{SN}=6$; in $\mathrm{FMN} 2^{-/-}$ , NSN=12, Trans=11, SN=26. (F) Control SRSF2-GFP droplet minute-scale 3D-dynamics $(50 \mu \mathrm{m} \mathrm{z-}$ projections) in Trans and SN cells highlighting random nucleoplasmic displacements and collisioncoalescence dynamics; droplet number and mean surface evolution indicated below in orange. (G) Nuclear SRSF2-GFP droplet collision-coalescence speed at the minute to hour-timescales. (Left) nuclear SRSF2-GFP droplet number evolution (in \% of initial droplet number) with Control, amplified (+Nocodazole), or disrupted cytoplasmic forces (+Taxol or FMN2 ${ }^{-/}$) in NSN oocytes; oocyte number, 4 oocytes for each condition; error bars represent mean \pm s.e.m.; $P$ values derived from two-tailed Wilcoxon matched-pairs signed rank tests, $* P=0.0195, * * P<0.0078$. (Right) nuclear droplet surface evolution between the initial timepoint $\left(t_{i}\right)$ and $t=96 \mathrm{~min}$; droplet number, Control $t_{i}=113, t_{96}=96$, Nocodazole $\mathrm{t}_{\mathrm{i}}=93, \mathrm{t}_{96}=58$, Taxol $\mathrm{t}_{\mathrm{i}}=114, \mathrm{t}_{96}=104, \quad F M N 2^{-/} \quad \mathrm{t}_{\mathrm{i}}=141, \mathrm{t}_{96}=124 ;$ violin plots with median \pm quartiles; $P$ values derived from two-tailed Mann-Whitney $U$-Tests, ns, not significant, $P>0.177, * * P=0.0071, * * * * P<0.0001$. (H) Nuclear droplet coalescence speed per hour relative to droplet number decrease in experimental NSN conditions (Control in blue, Nocodazole in green and $\mathrm{FMN}^{-/-}$in dark blue) and computational models with simulated NSN Control-like, Nocodazole-like, and $\mathrm{FMN}^{-/-}$-like cytoplasmic activity; error represents mean $\pm 95 \%$ confidence interval; number of experimental measurements, Control $\mathrm{NSN}=9$, Nocodazole $=9, \mathrm{FMN} 2^{-/}=7 ; 5$ simulations for each condition. (I) 3D-simulations ( $\mathrm{n}=41$ ) showing the stimulus-response relationship between cytoplasmic forces (intensities colored according to the gradient on the left) and nucleoplasmic SRSF2 droplet coalescence speed on an hour-to-day timescale; simulations start from an NSN-like nucleus state with 45 SRSF2 droplets; chromatin condensation and cytoplasmic activity intensification, simulating the transition into late growth, occurs at 12 hours. (J) Time frames of 3D-simulations in (I) with a gradient of starting-point cytoplasmic intensities that include the FMN2 $2^{-/}$-like and Control-like scenarios; SRSF2 droplets are in orange and a single grey nucleolus is depicted in a spherical nucleus-like container. Droplets in $\mathbf{A}, \mathbf{D}$, and $\mathbf{F}$ are outlined in dashed orange; color codes based on cytoplasmic stirring intensities; scale bars, $5 \mu \mathrm{m}$. 


\section{Figure 3}

A

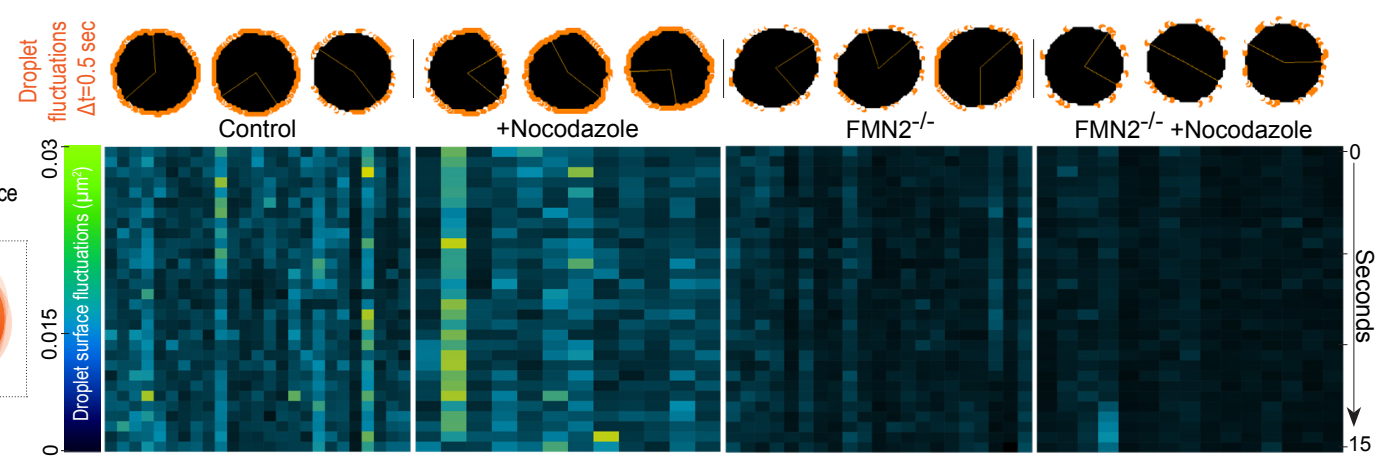

B

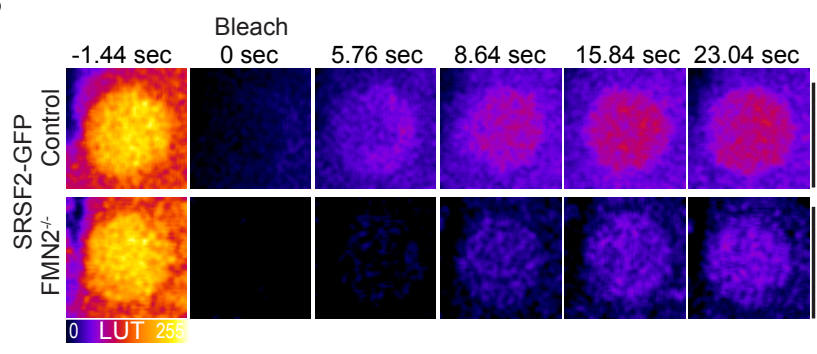

D

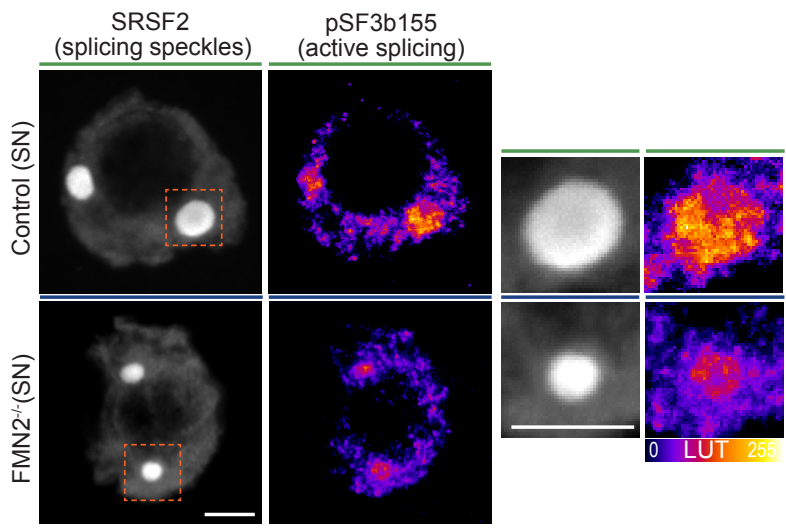

$\mathbf{F}$

mRNA exons in FMN2 ${ }^{-1-}$

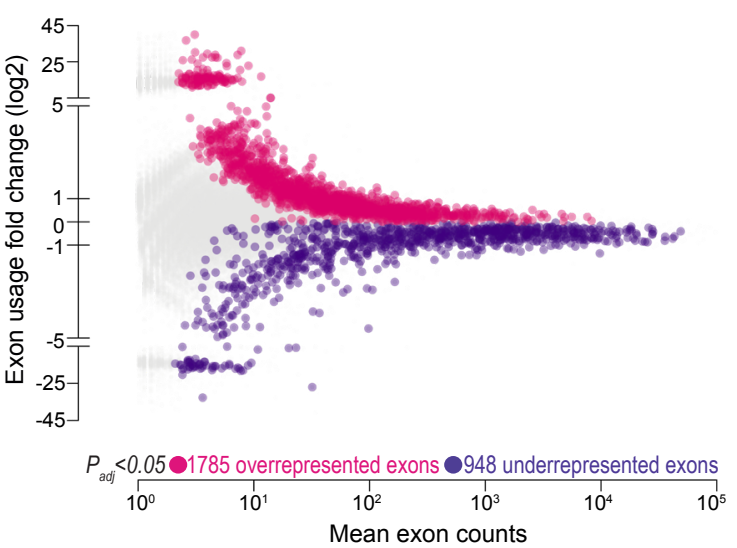

C
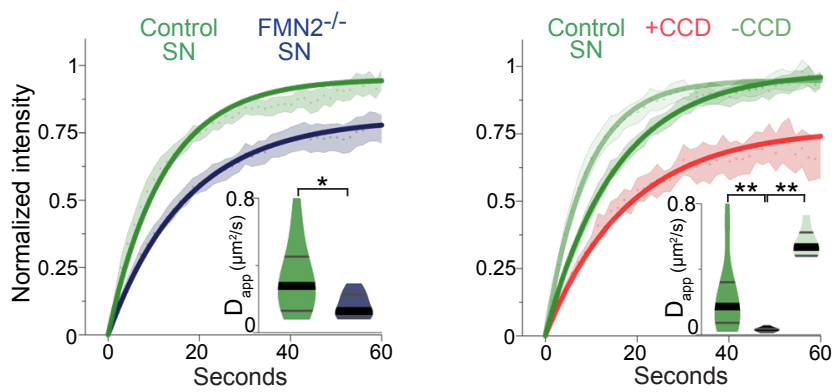

E

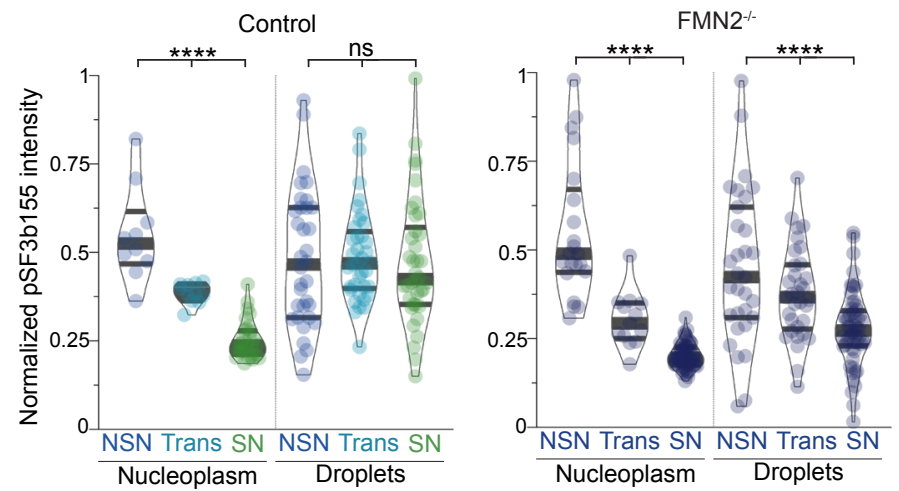

G

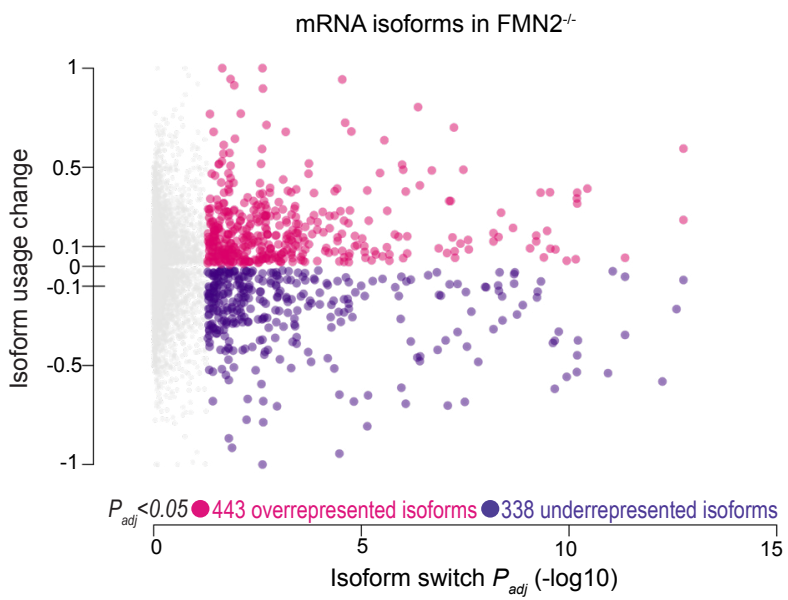


Fig. 3 | Cytoplasmic forces accelerate molecular-scale kinetics in nuclear liquid condensates and alter mRNA processing

(A) Scheme of droplet surface fluctuations as a function of cytoplasmic stirring (left); representative nuclear SRSF2-GFP droplet surface fluctuations (above right, droplet surface fluctuations in orange and droplet interiors are masked in black) and surface fluctuation intensity heatmaps of comparably sized droplets (below right; each column corresponds to a droplet and rows correspond to time) in SN oocytes with Control, amplified, or disrupted cytoplasmic forces; range of droplet radii, 2 to $2.7 \mu \mathrm{m}$; droplet number, Control=25; Nocodazole $=12 ; \mathrm{FMN}^{-/}=21 ; \mathrm{FMN} 2^{-/}+$Nocodazole $=15$. (B) Representative FRAP sequences of SRSF2-GFP droplets in Control and FMN2 $2^{-/-}$oocytes. (C) Normalized fluorescence intensity recovery curves (mean \pm s.e.m.) with simple exponential fits of SRSF2-GFP droplets in (left) Control and FMN2 $2^{-/-} \mathrm{SN}$ oocytes or in (right) Control, Cytochalasin-D treated $(+\mathrm{CCD})$, or CytochalasinD washout (-CCD) SN oocytes; insets, apparent diffusion coefficients $\left(D_{a p p}\right)$; droplet number, (left) Control=15, $\mathrm{FMN}^{-/}=9$; (right) Control $=12,+\mathrm{CCD}=7,-\mathrm{CCD}=6$; violin plots with median \pm quartiles; $P$ values derived from two-tailed Mann-Whitney $U$-Tests, ${ }^{*} P=0.0297$, ${ }^{* *} P<0.0052$. (D) Representative co-immunostainings of SRSF2 and phosphorylated SF3b155 (pT313; active splicing marker) in SN Control and FMN2 $2^{-/}$oocytes with higher magnifications of single droplets. (E) Quantifications of global nucleoplasmic or single droplet-specific pT313-SF3b155 intensities in Control and FMN2 $2^{-/-}$oocytes at the three growth stages; cell number, Control NSN=10, Trans $=11, \mathrm{SN}=32, \mathrm{FMN}^{-/-} \mathrm{NSN}=21$, Trans $=13$, $\mathrm{SN}=64$; Droplet number, Control $\mathrm{NSN}=34$, Trans $=37, \mathrm{SN}=38, \mathrm{FMN}^{-/} \mathrm{NSN}=31$, Trans $=33, \mathrm{SN}=56$; violin plots with median \pm quartiles; $P$ values derived from Kruskal-Wallis Tests, ns, not significant, $P=0.7786$, $* * * * P<0.0001$. (F-G) Differential mRNA exon usage (log2) versus mean abundance (mean exon counts) or differential mRNA isoform usage versus isoform switch $P_{a d j}(-\log 10)$ in SN FMN2 $2^{-/-}$ oocytes relative to Control SN FMN2 $2^{+/}$oocytes obtained with RNA-sequencing (from $\left({ }^{7}\right)$ ) and DEXSeq or IsoformSwitchAnalyzeR packages; Colored dots are significantly over/underrepresented (fuchsia/purple) exons or isoforms in FMN2 ${ }^{-/}$oocytes with a BH-adjusted (exons) or FDR-adjusted (isoforms) $P$ value $P_{a d j}<0.05$. Scale bars in $(\mathbf{B}, \mathbf{D}), 5 \mu \mathrm{m}$. 
bioRxiv preprint doi: https://doi.org/10.1101/2021.03.15.434387; this version posted March 16, 2021. The copyright holder for this preprint (which was not certified by peer review) is the author/funder. All rights reserved. No reuse allowed without permission.

\section{Figure 4}

A

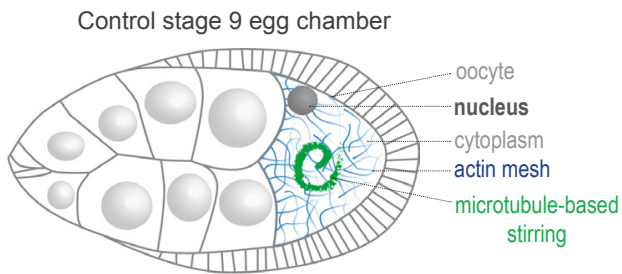

Cytoplasmic random stirring

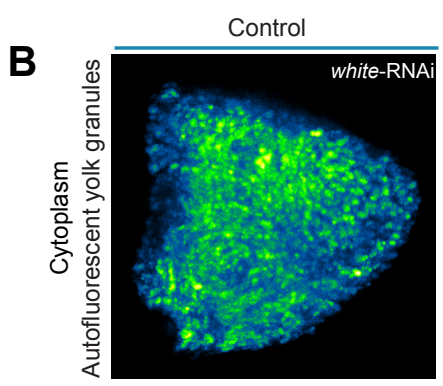

C

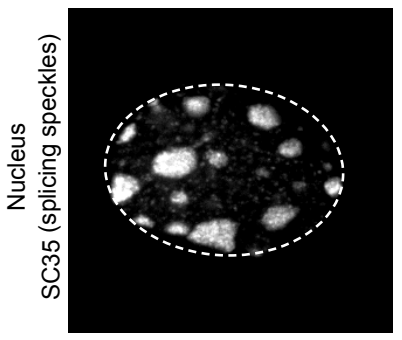

D

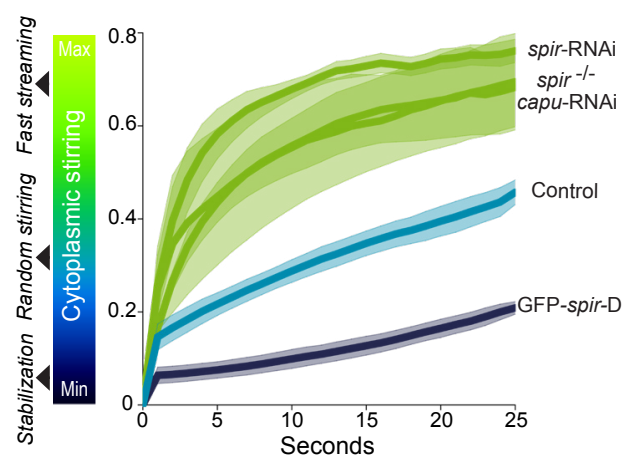

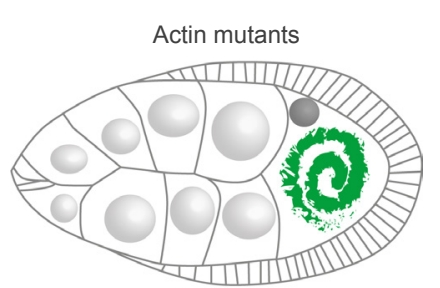

Cytoplasmic fast streaming
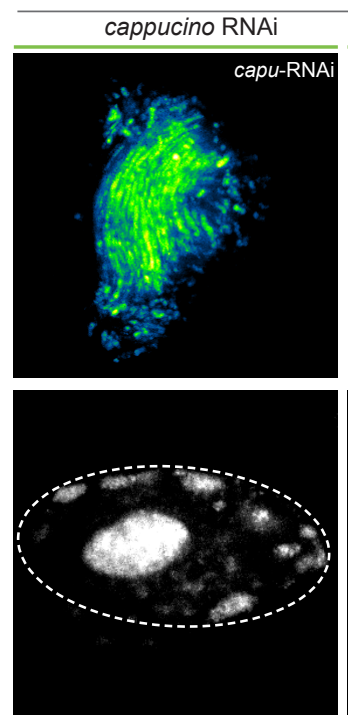

$\mathbf{E}$

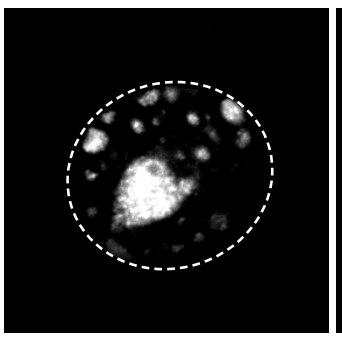

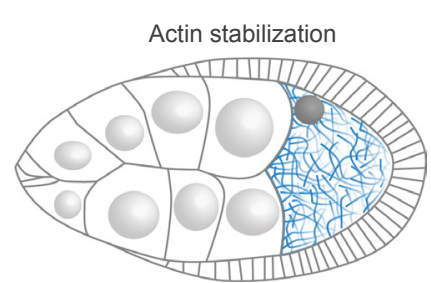

Cytoplasmic stabilization
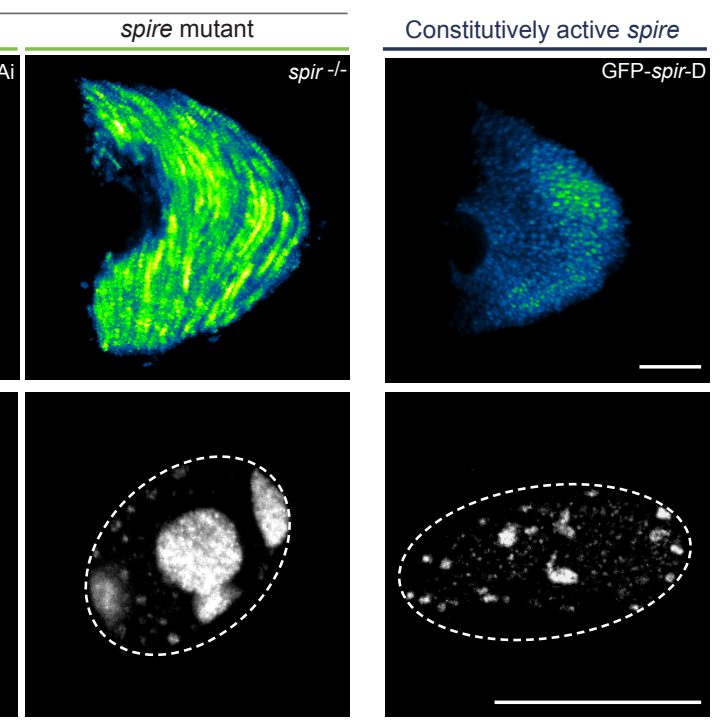
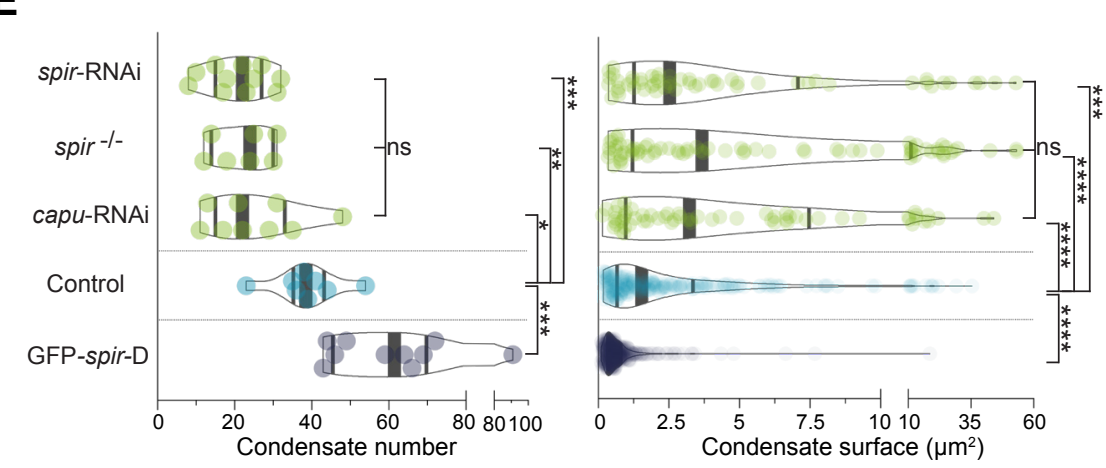
Fig. 4 | Cytoplasmic aptitude to drive subnuclear condensate reorganization is evolutionary conserved in insects.

(A) Illustrations of Drosophila melanogaster stage 9 egg chambers composed of the oocyte surrounded by nurse and follicular cells. At stage 9, cytoplasmic random stirring occurs in controls (left), premature microtubule-based fast streaming in actin mutants (center), and stabilization in mutants with constitutively active actin nucleators (right). (B) Time-projections of cytoplasmic stirring viewed with autofluorescent yolk granules in Control, cappuccino and spire mutants, or constitutively active spire overexpression mutants (GFP-spir-D); single z-slice time-projections of 25 seconds shown with 'GFB' LUTs. (C) Immunoreactivity of SC35 in nuclei of oocytes with Control cytoplasmic random stirring, amplified fast streaming, or stabilization; $2 \mu \mathrm{m}$ z-projections with nucleus regions outlined in dashed white. (D) Cytoplasmic stirring intensity measured in Control and mutant oocytes by image correlation analyses of cytoplasmic pixel evolution; cell number, Control=4, cappuccino $\mathrm{RNAi}=3$, spire $\mathrm{RNAi}=5$, spire $^{-/}=3$, GFP-spir- $D=2$; error bars represent mean \pm s.e.m. (E) Quantifications of SC35 condensate number and surface in nuclei of control and mutant oocytes; cell number, Control=8, cappuccino $\mathrm{RNAi}=9$, spire $\mathrm{RNAi}=11$, spire ${ }^{-/}=7$, GFP-spir $-D=10$; Condensate number, Control $=236$, cappuccino $\mathrm{RNAi}=62$, spire $\mathrm{RNAi}=55$, spire ${ }^{-/}=74$, GFP-spir $-D=437$; violin plots with median \pm quartiles; $P$ values derived from two-tailed Mann-Whitney $U$-Tests or Kruskal-Wallis Tests, ns, not significant, $P>0.576$, $* P=0.0121, * * P=0.0037, * * * P<0.001, * * * * P<0.0001$. Scale bars, $20 \mu \mathrm{m}$. 